\title{
Difficulties Experienced in Trauma Nursing Practice by Expert Emergency Nurses in Japan
}

\author{
Natsuko Makino1, Keiko Nakamura², Koji Ishikawa3 ${ }^{3}$, Miki Sugawara4 \\ ${ }^{1}$ Department of Nursing, School of Health Sciences, Sapporo Medical University, Sapporo, Japan \\ ${ }^{2}$ Graduate School of Nursing, Sapporo City University, Sapporo, Japan \\ ${ }^{3}$ Department of Nursing, Hokkaido University of Science, Sapporo, Japan \\ ${ }^{4}$ School of Nursing, Sapporo City University, Sapporo, Japan \\ Email:sky0122@sapmed.ac.jp
}

How to cite this paper: Makino, N., Nakamura, K., Ishikawa, K. and Sugawara, M. (2019) Difficulties Experienced in Trauma Nursing Practice by Expert Emergency Nurses in Japan. Open Journal of Nursing, 9, 1073-1087.

https://doi.org/10.4236/ojn.2019.910079

Received: September 30, 2019

Accepted: October 28, 2019

Published: October 31, 2019

Copyright $\odot 2019$ by author(s) and Scientific Research Publishing Inc. This work is licensed under the Creative Commons Attribution International License (CC BY 4.0).

http://creativecommons.org/licenses/by/4.0/ (c) (i) Open Access

\begin{abstract}
The objective of this study was to determine the difficulties experienced in trauma nursing practice by expert emergency nurses in Japan. Ten nurses who worked at emergency and critical care centers in Japan were directly involved in nursing patients were selected by purposive sampling and underwent semi-structured interviews regarding the difficulties they experienced in trauma nursing. The nurses were specialists in critical care nursing or were certified emergency nurses. The interview responses were analyzed by the qualitative descriptive method. The following six categories of difficulties associated with the direct care of trauma patients and their family members encountered in trauma nursing practice were identified: [Initial handling of highly urgent trauma patients], [Understanding and observing the condition of patients who are in the treatment stage], [Judging when to transition to end-stage care for patients difficult to save], [Mitigating suffering and expanding safe activities of daily living for trauma patients], [Transitional care with a long-term perspective for trauma patients], and [Relationships with the family members of patients who died]. Three categories of difficulties related to the role of the expert nurse in trauma nursing practice were identified: [Person-to-person coordination and cooperation for a trauma patient], [Playing an educational role for the nursing staff], and [Pursuing expertise in trauma nursing and gaining empirical knowledge]. The findings suggested a need to establish systems for training and consultative support and opportunities to create meaning by reflecting on fulfillment and nursing practice.
\end{abstract}

\section{Keywords}

Trauma Nursing Practice, Difficulties, Expert Nurse, Emergency Care 


\section{Introduction}

The number of deaths from physical trauma such as traffic accidents is decreasing from year to year in Japan. Accidents such as traffic accidents are the main cause of death in children and adolescents [1]. To prevent such deaths from external causes, the Japan Nursing for Trauma Evaluation and Care (JNTEC ${ }^{\mathrm{TM}}$ ) guidelines intended for nurses have been promulgated in Japan, and many nurses involved in emergency nursing have learned trauma nursing.

Several studies of trauma nursing in Japan have been reported. For example, a study employing participant observations and interviews of critically injured patients found that such patients are overwhelmed by sudden shock during recovery, fear having relationships severed, are intent on escaping from their extreme situation, and make efforts to understand the event that occurred [2]. A study that focused on the needs of relatives of head trauma patients found that the family members' needs included information and emotional support [3]. Additionally, a case report [4] and a study [5] about the educational effect of trauma nursing are reported. However, few studies have examined what constitutes trauma nursing practice in Japan. Investigators, therefore, conducted interviews of emergency nurses, with a focus on trauma nursing practice. They reported that emergency nurses see the trauma nursing practice according to a timeline from the initial trauma to the patient's reintegration into society and that the nursing can be classified into three categories: nursing care for the trauma patient, nursing care for the members of the trauma patient's family, and nursing care as a trauma care team [6]. The study results showed that emergency nurses required a broad range of knowledge and skills for trauma nursing practice and that such practice is wide-ranging.

Emergency nurses experience vast difficulties. In addition to providing direct care to patients and relatives of patients whose status is complicated and highly urgent and patients in critical condition, they must coordinate across disciplines. It is surmised that trauma care nursing in particular is difficult even for expert nurses with years of experience, due to factors such as the diversity and complexity of the care and time pressures. A survey of previous studies of the difficulties encountered by emergency nurses in Japan found that the topics examined included the relationships between patients in critical condition and their family members [7], end-stage care [8] [9], support for decision-making by patient representatives [10], and matters related to family members [11]. Focusing on the difficulties associated with trauma nursing, the feelings nurses have experienced in caring for trauma patients include fear and discouragement [12]. In a survey of nurses working in emergency outpatient care regarding initial emergency responses, $46.8 \%$ responded that they "somehow managed" to care for trauma patients, and $4.7 \%$ said they were "unable to care for such patients well". The most common response was that a problem they encountered in dealing with such patients was that they "lacked the necessary knowledge and skills" [13]. 
Thus, although the difficulties associated with trauma nursing have been identified sporadically, the substantive difficulties that nurses experience and the background factors that contribute to such difficulties have not been adequately identified. To address the diverse difficulties experienced, combining knowledge and experience to reexamine one's own emotions and behaviors objectively and giving meaning to nursing care have been found to be important [14]. Consequently, determining the substance of the difficulties that emergency nurses experience in trauma nursing practice and the background of such difficulties may make it possible to integrate trauma nursing knowledge and experience and give meaning to such nursing. Moreover, it may provide basic data needed to build a training program in trauma nursing.

Therefore, the objective of this study was to identify the difficulties experienced in trauma nursing practice by expert emergency nurses in Japan.

\section{Methods}

\subsection{Definitions of Terms}

1) Trauma nursing practice

Nursing whose main duties consist of acting directly and indirectly on patients and their family members, from resuscitation to rehabilitation, to facilitate recovery with regard to existing or potential health problems resulting from trauma.

2) Expert nurse

A nurse who is certified by the Japanese Nursing Association and implements superior, advanced nursing practice. The nurses in this study were certified as Certified Nurse Specialists in critical care nursing or as Certified Nurses in emergency nursing by the Japanese Nursing Association.

3) Difficulties

Difficulties are described as experiences of aggravation, unpleasantness, anxiety, or distress encountered by expert emergency nurses during the course of nursing practice for trauma patients and their family members.

\subsection{Design}

A qualitative descriptive design was used in this study to identify the difficulties experienced in trauma nursing practice by expert emergency nurses in Japan. Qualitative descriptive designs are used in qualitative research for studies that are descriptive in nature, particularly for examining health care and nursing-related phenomena.

\subsection{Requirements for Selection as a Subject}

The 10 nurses selected as subjects met all of the following requirements: 1) working as a nurse and directly involved in nursing patients at the time of selection; 2) working at an emergency and critical care center certified by the Japan Association for Acute Medicine; 3) involved in nursing at least 10 trauma pa- 
tients per year; 4) certified as a Certified Nurse Specialist in critical care nursing or as a Certified Nurse in emergency nursing by the Japanese Nursing Association, with the nurse's name and institution published on the association's website; and 5) not a nursing supervisor or manager. The selection requirements took into account the fact that most trauma patients in Japan are transported to emergency and critical care centers, although this depends on the severity of their injuries, and the fact that most of the expert emergency nurses in Japan are Certified Nurse Specialists in critical care nursing or Certified Nurses in emergency nursing.

\subsection{Data Collection}

The data were collected from March 2017 to July 2017.

After being selected through a purposive sampling method using the Japanese Nursing Association website, the subjects were given a written explanation of aspects of the study, such as its purpose and the ethical considerations involved, and were asked whether they wanted to participate in the study. This correspondence was carried out by postal mail. If the subject wished to participate in the study, consent was obtained from the nursing supervisor of their institution following the same method. Semi-structured interviews were conducted at a location where privacy could be ensured. Each subject was interviewed only once by an investigator and the interview took approximately 45 to 60 minutes. The topics covered in the interview were basic attributes (sex, years of nursing experience, years of emergency nursing experience, department with which subject was currently affiliated) and the difficulties experienced by the subject in trauma nursing practice. The interview was recorded with the consent of the subject.

\subsection{Data Analysis}

The data were analyzed by qualitative descriptive analysis, which comprised an analysis by subject and an overall analysis. First, a verbatim transcript was prepared from the data, and the transcript was read repeatedly and carefully to gain an overview of the content. Next, contextual units were extracted from the verbatim transcript for inclusion in the analysis by subject, focusing on the difficulties experienced by the subject in trauma nursing practice. An initial summary was prepared in a manner that preserved the semantic content of the individual contextual units. The level of abstraction of the expressions that reflected that description was then increased to prepare a secondary summary. In the overall analysis, the secondary summaries obtained for all of the subjects were classified based on their similarities and commonalities, and subcategories and categories were formulated and labeled.

\subsection{Reliability Assurance}

To examine the rigor and accuracy of the analysis results, a trial interview was conducted with one expert nurse who met the inclusion requirements. This re- 
sulted in improvements in the interviewer's interviewing skills and the accuracy of the interview guide. In addition, member checking was performed, and a determination of whether the interpretation of the data was valid was requested.

Throughout the process, the investigators consulted with one another regarding data extraction and the formulation and labeling of categories. In addition, an investigator who specialized in emergency nursing and was experienced in qualitative research provided supervision with regard to the analysis results.

\subsection{Ethical Considerations}

This study was conducted with approval from the Ethics Committee of Sapporo Medical University (Approval No. 28-2-61). The participating institution and subjects were given written and oral explanations of aspects of the study such as the following: the intent and purpose of the study; that study participation was voluntary; that they could decline to participate; that anonymity and confidentiality would be maintained; that rigorous methods of data handling, retention, and disposal would be employed; the psychological burden associated with recalling past experiences and how to cope with it; and how the results would be published. Written informed consent to participate was obtained from all subjects.

\section{Results}

\subsection{Overview of the Subjects}

There were 10 subjects (three men and seven women) (Table 1) with a mean nursing experience of $19.7 \pm 6.0$ years, a mean working experience in an emergency and critical care center of $15.2 \pm 5.5$ years, and a mean involvement in trauma nursing of $15.2 \pm 5.5$ years. All subjects met the inclusion criteria as described above.

\subsection{Difficulties Experienced in Trauma Nursing Practice by Expert Emergency Nurses in Japan}

The following nine categories were extracted for the difficulties experienced in trauma nursing practice by expert emergency nurses in Japan: [Initial handling of highly urgent trauma patients], [Understanding and observing the condition of patients who are in the treatment stage], [Judging when to transition to end-stage care for patients difficult to save], [Mitigating suffering and expanding safe activities of daily living (ADLs) for trauma patients], [Transitional care with a long-term perspective for trauma patients], [Relationships with the family members of patients who died], [Person-to-person coordination and cooperation for a trauma patient], [Playing an educational role for the nursing staff], and [Pursuing expertise in trauma nursing and gaining empirical knowledge]. In Figure 1, each category and subcategory is sorted into two time periods: "acute stage of injury" and "post-early stage of injury through rehabilitation".

The categories are explained below. Categories, subcategories, and typical 
Table 1. General information about participants.

\begin{tabular}{|c|c|c|c|c|c|c|c|}
\hline ID & Sex & $\begin{array}{l}\text { Age, } \\
\text { Years }\end{array}$ & $\begin{array}{l}\text { Nursing } \\
\text { Experience, } \\
\text { Years }\end{array}$ & $\begin{array}{c}\text { Experience in } \\
\text { Emergency } \\
\text { Nursing } \\
\text { Care, Years }\end{array}$ & $\begin{array}{c}\text { Nursing } \\
\text { Experience } \\
\text { in Trauma } \\
\text { Care, Years }\end{array}$ & Department & Certification \\
\hline A & Male & Late $30 \mathrm{~s}$ & 12 & 10 & 10 & $\begin{array}{c}\text { Emergency and } \\
\text { critical care Center }\end{array}$ & $\begin{array}{l}\text { Certified Nurse in } \\
\text { emergency nursing }\end{array}$ \\
\hline B & Female & Late $30 \mathrm{~s}$ & 15 & 11 & 11 & $\begin{array}{l}\text { Emergency and } \\
\text { critical care Center }\end{array}$ & $\begin{array}{l}\text { Certified Nurse in } \\
\text { emergency nursing }\end{array}$ \\
\hline $\mathrm{C}$ & Female & Early 40 s & 21 & 16 & 16 & $\begin{array}{l}\text { Emergency and } \\
\text { critical care Center }\end{array}$ & $\begin{array}{l}\text { Certified Nurse in } \\
\text { emergency nursing }\end{array}$ \\
\hline $\mathrm{D}$ & Female & Early 40 s & 17 & 13 & 13 & $\begin{array}{l}\text { Emergency and } \\
\text { critical care Center }\end{array}$ & $\begin{array}{l}\text { Certified Nurse in } \\
\text { emergency nursing }\end{array}$ \\
\hline $\mathrm{E}$ & Female & Late $40 \mathrm{~s}$ & 25 & 8 & 8 & $\begin{array}{l}\text { Emergency and } \\
\text { critical care Center }\end{array}$ & $\begin{array}{l}\text { Certified Nurse in } \\
\text { emergency nursing }\end{array}$ \\
\hline $\mathrm{F}$ & Male & Late $30 \mathrm{~s}$ & 10 & 10 & 10 & $\begin{array}{l}\text { Emergency and } \\
\text { critical care Center }\end{array}$ & $\begin{array}{l}\text { Certified Nurse Specialist } \\
\text { in critical care nursing }\end{array}$ \\
\hline G & Female & Early 40s & 21 & 19 & 19 & $\begin{array}{l}\text { Emergency and } \\
\text { critical care Center }\end{array}$ & $\begin{array}{l}\text { Certified Nurse Specialist } \\
\text { in critical care nursing }\end{array}$ \\
\hline $\mathrm{H}$ & Male & Early 40 s & 22 & 21 & 21 & $\begin{array}{c}\text { Emergency and } \\
\text { critical care Center }\end{array}$ & $\begin{array}{l}\text { Certified Nurse Specialist } \\
\text { in critical care nursing }\end{array}$ \\
\hline I & Female & Late $40 \mathrm{~s}$ & 27 & 23 & 23 & $\begin{array}{l}\text { Emergency and } \\
\text { critical care Center }\end{array}$ & $\begin{array}{l}\text { Certified Nurse Specialist } \\
\text { in critical care nursing }\end{array}$ \\
\hline $\mathrm{J}$ & Female & Late $40 \mathrm{~s}$ & 27 & 21 & 21 & $\begin{array}{l}\text { Emergency and } \\
\text { critical care Center }\end{array}$ & $\begin{array}{l}\text { Certified Nurse Specialist } \\
\text { in critical care nursing }\end{array}$ \\
\hline
\end{tabular}

statements are enclosed in [ ], (), and \{\}, respectively.

1) [Initial handling of highly urgent trauma patients]

This category indicates the difficulty of the initial handling of trauma patients when they are first brought to the hospital. The difficulty of the initial response, which affects whether the patient lives or dies and their prognosis, was indicated by subcategories such as the following: (I understand that the response that the patient receives when they are brought to the hospital will affect their prognosis, and this makes it difficult to handle highly urgent treatments) and (Responding is difficult because we are pressed to quickly make decisions for trauma patients in life-or-death situations). In addition, the subjects felt it was difficult to provide the wide range of responses required, such when a sudden change occurs during treatment of a trauma patient (It is difficult to switch responses due to differences in the patient's condition as a result of a sudden change) or when observing extensive sites of trauma (Responding to extensive injuries requires knowledge and observational ability, and I feel this is difficult). Moreover, the subjects said that nurses are assigned multiple duties when a trauma patient is brought to the hospital and that responding is difficult under those circumstances (Handling multiple duties is difficult when a trauma patient is brought to the hospital). 


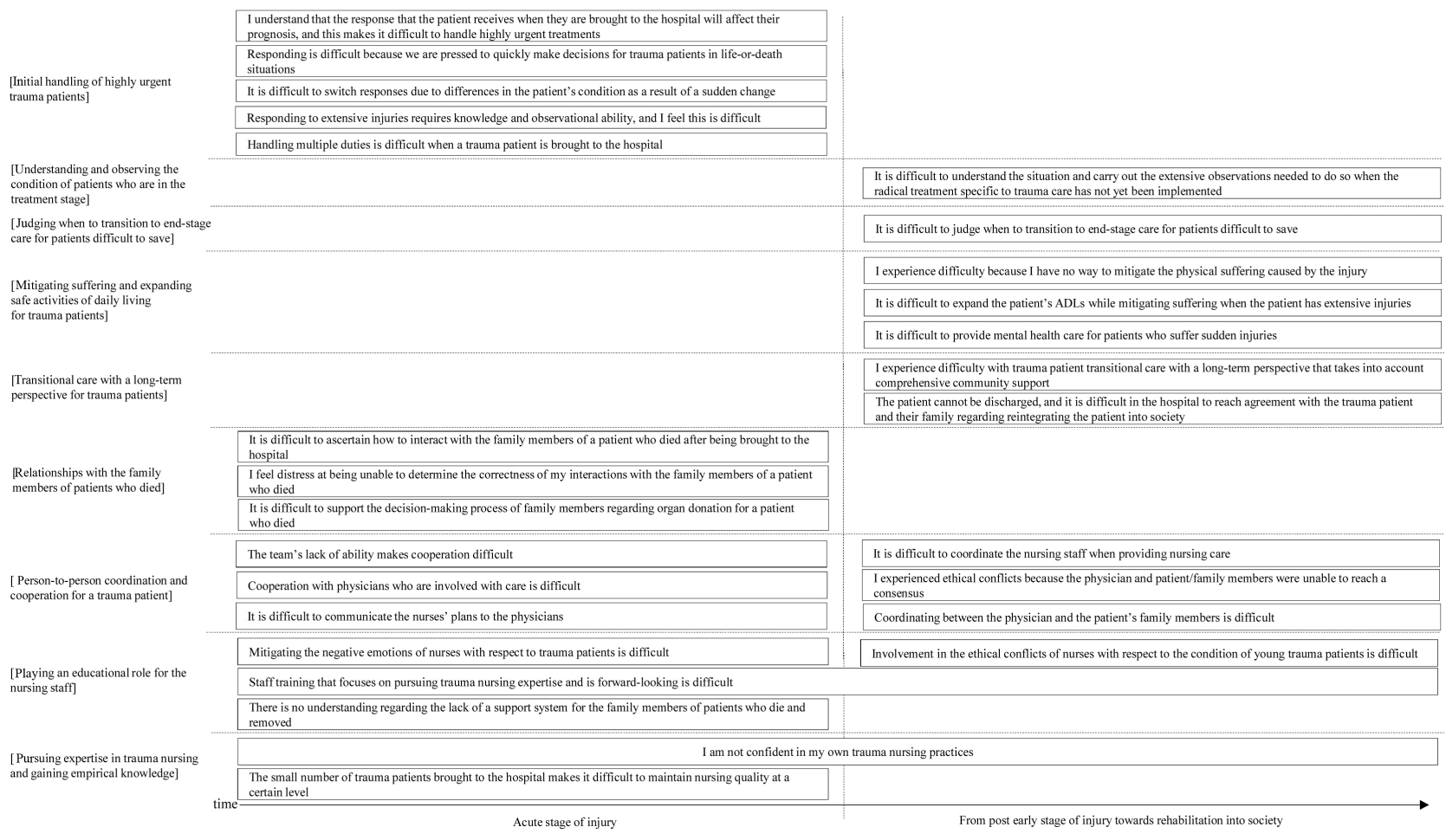

Figure 1. Difficulties in trauma nursing as experienced by expert nurses working in the emergency care field in Japan.

2) [Understanding and observing the condition of patients who are in the treatment stage]

This category indicates difficulties associated with understanding and observing the treatment process for trauma patients for whom the aim is complete recovery after going through the treatment stage specific to trauma. The nurses experienced difficulty with the fact that a stepwise treatment plan intended to provide damage control specific to trauma care requires the nurse to ascertain the situation at each treatment stage and carry out extensive observations at each stage (It is difficult to understand the situation and carry out the extensive physical observations needed to do so when the radical treatment specific to trauma care has not yet been implemented).

3) [Judging when to transition to end-stage care for patients difficult to save]

This category indicates the difficulty of judging when to transition from aggressive treatment to end-stage care for patients difficult to save. Typical statements by subjects were as follows: \{It is difficult to judge how extensively to treat a patient who appears difficult to save when they are first brought to the hospital\}, (It is difficult to judge when to transition to end-stage care for patients difficult to save).

4) [Mitigating suffering and expanding safe ADLs for trauma patients]

This category indicated the difficulty of mitigating the physical and mental suffering associated with injury and expanding the patient's ADLs. Subjects described the difficulty of finding how to mitigate the physical suffering associated with traumatic injury (I experience difficulty because I have no way to mitigate 
the physical suffering caused by the injury). In addition, they described the difficulty of expanding the ADLs of patients who are suffering (It is difficult to expand the patient's ADLs while mitigating suffering when the patient has extensive injuries) and of providing mental health care for trauma patients (It is difficult to provide mental health care for patients who suffer sudden injuries).

5) [Transitional care with a long-term perspective for trauma patients]

This category indicates the difficulty of providing transitional care with a view to the trauma patient's reintegration into society. Subjects described the difficulty of being unable to attempt a hospital transfer or discharge of trauma patients transported to an acute-care hospital (I experience difficulty with trauma patient transitional care with a long-term perspective that takes into account comprehensive community support). In addition, they described difficulty in reaching agreement with the patient and their family on reintegrating the patient into society (The patient cannot be discharged, and it is difficult in the hospital to reach agreement with the trauma patient and their family regarding reintegrating the patient into society).

6) [Relationships with the family members of patients who died]

This category indicates the difficulty of the relationship with the family members of a patient who died soon after trauma. Subjects described feeling distress at being unable to decide based on introspection if their interactions with a patient who died were appropriate (It is difficult to ascertain how to interact with the family members of a patient who died after being brought to the hospital) and (I feel distress at being unable to determine the correctness of my interactions with the family members of a patient who died). In addition, because it is not unusual for brain death to occur in trauma patients, they described difficulty in helping the family members of the patients make a decision about organ donation (It is difficult to support the decision-making process of family members regarding organ donation for a patient who died).

7) [Person-to-person coordination and cooperation for a trauma patient]

This category indicates the difficulty of coordination and cooperation among physicians, nurses, and the family members of trauma patients during treatment. Subjects described difficulties in team cooperation with regard to treatment (The team's lack of ability makes cooperation difficult) and in cooperating with physicians (Cooperation with physicians who are involved with care is difficult) and (It is difficult to communicate the nurses' plans to the physicians). In addition, subjects described difficulty in intradisciplinary staff coordination because ensuring the safety of the injury sites in trauma patients is difficult for one nurse alone and requires the care of several nurses (It is difficult to coordinate the nursing staff when providing nursing care). In addition, subjects described difficulty in coordinating between the medical staff and the patients and their family members (I experienced ethical conflicts because the physician and patient/family members were unable to reach a consensus) and (Coordinating between the physician and the patient's family members is difficult). 
8) [Playing an educational role for the nursing staff]

This category indicates the difficulty of training nursing staff in trauma nursing practice, coordination, and ethics. Subjects described difficulties arising from ethical conflicts and negative emotions on the part of the nursing staff with respect to patients (Involvement in the ethical conflicts of nurses with respect to the condition of young trauma patients is difficult) and (Mitigating the negative emotions of nurses with respect to trauma patients is difficult). Subjects experienced difficulties related to practical training that emphasizes the pursuit of expertise and is forward-looking (Staff training that focuses on pursuing trauma nursing expertise and is forward-looking is difficult). They also experienced difficulty in obtaining the understanding of those around them in carrying out their role as expert nurses (There is no understanding regarding the lack of a support system for the family members of patients who die).

9) [Pursuing expertise in trauma nursing and gaining empirical knowledge]

This category indicates the difficulties encountered by the nurses themselves in obtaining training and empirical knowledge of trauma nursing practice, coordination, and ethics and pursuing expertise for the nursing staff. Subjects said they were not confident in the practices they implemented (I am not confident in my own trauma nursing practices). In addition, they said that the small number of trauma patients transported to the emergency and critical care center prevented them from accumulating experience (The small number of trauma patients brought to the hospital makes it difficult to maintain nursing quality at a certain level).

\section{Discussion}

\subsection{Difficulties Related to the Direct Care of Trauma Patients and Their Family Members Encountered in Trauma Nursing Practice}

Of the nine categories described above, the first six are specific to those that arise in trauma care associated with the direct care of trauma patients and their family members encountered mainly in trauma nursing practice: [Initial handling of highly urgent trauma patients], [Understanding and observing the condition of patients who are in the treatment stage], [Judging when to transition to end-stage care for patients difficult to save], [Mitigating suffering and expanding safe ADLs for trauma patients], [Transitional care with a long-term perspective for trauma patients], and [Relationships with the family members of patients who died]. The characteristics of these categories are discussed below.

Trauma nursing requires that highly urgent treatment be provided with limited information, and the care provided after the person's life is saved is also complicated. The trauma patients with whom the subjects were involved in this study included patients who were near death and patients who were treated in a stepwise fashion with the aim of full recovery. The subjects' statements regarding these patients fell into the following subcategories: (It is difficult to respond 
quickly under time pressure because early decisions and treatment for the sudden and varied traumatic injuries determine the prognosis) and (The need for extensive observations that provide an understanding of the situation is a difficulty because the patient enters the intensive care unit without having received radical treatment specific to traumatic injuries). In trauma care, damage control focused on preventing failure of the patient's physiological signs is a more common treatment strategy than one focused on repairing injuries. Consequently, the subjects must observe the patient while determining the stage of damage control that he or she is at and, at the same time, provide nursing care aimed at saving a patient who is wavering between life and death. This diversity makes it difficult to provide nursing care, and this is characteristic of the difficulties nurses face in trauma nursing practice.

At the same time, the subjects mentioned "judging when to transition to end-stage care for patients difficult to save" as a difficulty. This suggested a conundrum in which the subjects needed to have the perspective of saving the patient's life and the perspective of preparing for end-stage care when implementing nursing practices during treatment. The difficulty of changing gears in this way has been studied in areas such as cancer nursing, and the need to increase the preparedness of the patients and their family members has been discussed [15]. However, trauma patients are often injured suddenly in an event such as a traffic accident or airplane crash [Relationships with the family members of patients who died], and in many cases, they cannot be saved. Consequently, preparedness for the death on the part of the patient or family members was lacking, and this may have made nursing care more difficult.

Next, the subjects indicated that it was difficult to ensure the safety and comfort of trauma patients while expanding their ADLs [Mitigating suffering and expanding safe ADLs for trauma patients]. Trauma patients often have tissue damage as a result of their injuries. On the other hand, beginning rehabilitation early has been shown to play a role in the improvement in the patient's subsequent functional prognosis. Pain has been reported to be the stressor most commonly experienced by trauma patients [16], and the pain was a typical source of suffering in the present study. With traumatic injuries, it is difficult to treat conditions such as progression of the inflammation and necrosis that frequently occur, despite treatment, as a result of latent infection or bruising, and this prolongs the patient's suffering [2]. While mitigating the suffering of the patients, the subjects must make arrangements so that support for recovery is continued, and these responsibilities make assessments difficult.

Lastly, [Transitional care with a long-term perspective for trauma patients] is discussed. The patients with which the subjects in the present study were involved included patients who had difficulty reintegrating into society after their traumatic injury. Various factors prevent a patient who has undergone severe trauma from sitting up or getting out of bed during the acute phase, and because there is residual impairment, the patient's life will be completely different as 
compared with before the injury. Because the subjects in this study possessed empirical knowledge accumulated through nursing trauma patients, they anticipated the patients' reintegration into society beginning from the acute phase and therefore understood the patients. This may be why the subjects acutely felt the difficulty of a smooth transition during the patient's reintegration into society.

\subsection{Difficulties Concerning the Role of the Expert Nurse in Trauma Nursing Practice}

The following three categories mainly concerned the role of the expert nurse in trauma nursing practice: [Person-to-person coordination and cooperation for a trauma patient], [Playing an educational role for the nursing staff], and [Pursuing expertise in trauma nursing and gaining empirical knowledge]. The characteristics of these categories are discussed below.

The role of the emergency nurse in trauma nursing practice has been found to be not only to provide care for the patient and their family but also to coordinate across disciplines as a member of the trauma care team [6]. Subjects in the present study mentioned [Person-to-person coordination and cooperation for a trauma patient] as a category of difficulty. In the emergency medicine setting, there is very little discussion among coworkers regarding their understanding of phenomena that occur or the actions to be taken, and care is carried out under an assumption of mutual understanding [17]. For trauma patients, initial trauma care that adheres to a systematic treatment strategy is recommended, and multiple treatments are administered at the same time. This likely requires the emergency nurse to exhibit an ability to coordinate combined with foresight. Although the subjects in the present study were experienced and skilled, they still experienced difficulty in coordinating across disciplines. This indicates that the diversity of trauma in terms of mechanisms of injury, severity, and disease types likely played a role in this difficulty. It is also related to the subcategory. (The small number of trauma patients brought to the hospital makes it difficult to maintain nursing quality at a certain level) of the category [Pursuing expertise in trauma nursing and gaining empirical knowledge].

The category [Playing an educational role for the nursing staff] is discussed next. The subjects in this study played an educational role in their organization, and they experienced difficulties with respect to supporting the emotional reactions of nurses prompted by the mechanisms of injury in trauma [Mitigating the negative emotions of nurses with respect to trauma patients is difficult]. Nursing is an emotional occupation and one that is affected by the responses of the patients. Nurses experience negative emotions in response to events such as accidents that are the fault of the trauma patients themselves, and the subjects' statements described the difficulty of providing training for those cases. The fact that the subjects practiced skilled nursing likely explains this difficulty as it pertains to areas such as support for the family members of patients who die, and 
coordination with family members of patients with little chance of recovery, because these are types of care that the subjects consider important.

\subsection{Implications for Nursing Practice}

Possible responses to the difficulties experienced in trauma nursing practice by expert emergency nurses in Japan are described below. Trauma centers like those in Europe and the United States do not exist in Japan due to problems in establishing an emergency medicine system. Consequently, trauma patients in serious condition are transported to emergency and critical care centers. Emergency nurses must therefore handle patients with a wide range of problems, from trauma patients to patients with cardiopulmonary arrest caused by intrinsic factors, which gives rise to difficulties such as those indicated by the categories: [Initial handling of highly urgent trauma patients], [Understanding and observing the condition of patients who are in the treatment stage], and [Pursuing expertise in trauma nursing and gaining empirical knowledge]. Skill in an area is generally field-specific, and transferring that skill to a different field is considered difficult [18]. Even a nurse with good knowledge of emergency nursing would likely have difficulty exhibiting the same level of ability for patients/family members in the specialized field of trauma as they would for other patients. This suggests the need for thorough training in trauma nursing.

Next, the subjects said they experienced difficulty with end-stage nursing, as indicated by the categories [Judging when to transition to end-stage care for patients difficult to save] and [Relationships with the family members of patients who died]. Although deaths resulting from trauma peak with the passage of time, an instant death or death that occurs within several hours after injury at the scene of the injury is not expected by the patient or their family and therefore poses a crisis. The difficulties of judging when to transition from lifesaving treatment to end-stage care and responding to a situation in which a patient's death is not accepted by their family members have previously been reported [19], and the results were similar to those of the present study. To resolve these difficulties, it may be necessary to take measures such as establishing a consultative support system for difficult cases or consulting with a nurse who specializes in family nursing.

In addition, the subjects in this study were required to fulfill a variety of roles, as indicated by the categories [Playing an educational role for the nursing staff] and [Person-to-person coordination and cooperation for a trauma patient]. Although the subjects were qualified and therefore fulfilled these roles, the results indicated that this was a source of difficulty. Introspection and reflection by nurses fosters a conceptual ability for nursing practice [20], which indicates that creating opportunities to make nursing meaningful is a way of resolving such difficulties.

Lastly, we discuss difficulties in taking a long-term perspective, as indicated by the category [Transitional care with a long-term perspective for trauma pa- 
tients]. In Japan, comprehensive community care is well developed, and this requires nursing development that anticipates the transition from the acute phase to reintegration into society. For trauma patients, the functional disability that remains after the injury makes reintegration into society difficult. Moreover, many trauma patients are young, which creates major challenges to their reintegration into society. Expert nurses like the subjects of this study can bring about a resolution of these difficulties by maintaining a long-term perspective, assuming responsibility for the patient's condition, and encouraging utilization of the patients' family members, those close to the patients, and the societal resources of the community.

\section{Study Limitations}

The subjects in this study were limited to expert emergency nurses who filled the selection criteria. Moreover, there were only 10 subjects in the study. Consequently, the results obtained represented the subjects' portion of a wide range of difficulties experienced. Moreover, the departments with which the subjects were affiliated covered a broad range from initial trauma care to care subsequent to the initial trauma. Therefore, differences in their trauma nursing experience cannot be ruled out. Challenges for the future will be to increase the sample size, identify the variety of difficulties that take into account the backgrounds of the subjects, and construct a system for resolving the difficulties experienced in trauma nursing practice.

\section{Conclusion}

For the difficulties experienced in trauma nursing practice by expert emergency nurses in Japan, the following six categories of difficulties associated with the direct care of trauma patients and their family members encountered in trauma nursing practice were identified: [Initial handling of highly urgent trauma patients], [Understanding and observing the condition of patients who are in the treatment stage], [Judging when to transition to end-stage care for patients difficult to save], [Mitigating suffering and expanding safe ADLs for trauma patients], [Transitional care with a long-term perspective for trauma patients], and [Relationships with the family members of patients who died]. Three categories of difficulties related to the role of the expert nurse in trauma nursing practice were identified: [Person-to-person coordination and cooperation for a trauma patient], [Playing an educational role for the nursing staff], and [Pursuing expertise in trauma nursing and gaining empirical knowledge]. The findings suggested need to establish systems for training and consultative support and opportunities to create meaning by reflecting on fulfillment and nursing practice.

\section{Acknowledgements}

We are deeply grateful to the nursing supervisors and nurses of the institution that participated in this study. This work was supported by the Japan Society for 
the Promotion of Science (JSPS) KAKENHI under grant number JP16K20755.

\section{Conflicts of Interest}

The authors declare no conflicts of interest regarding the publication of this paper.

\section{References}

[1] Health, Labour and Welfare Statistics Association (2018) Volume 2. Eiseinoshuyoushihyou Jikoudoutai. Health, Labour and Welfare Statistics Association (Volume). Journal of Hearth and Welfare Statistics, 63, 62-70.

[2] Sasaki, Y., Inoue, T., Yatomi, Y. and Suzuki, K. (2007) Process of Situation Recognition and Adaptation in the Recovery Phase of Critically Injured Patients. Journal of Japanese Association for Emergency Nursing, 8, 22-31.

[3] Iwai, S., Ishikawa, H., Hatanaka, T. and Taniguchi, K. (2005) Family Needs of Patients with Acute Phase Traumatic Brain Injury. Journal of Japanese Association for Emergency Nursing, 6, 23-29.

[4] Kimura, K. (2005) Consideration of Nursing of Pulmonary Complication Prevention to a Chest Traumatic Patient. Emergency Care, 18, 495-498.

[5] Masuyama, J., Yamaguchi, M. and Hiroshima, Y. (2007) Traumatic Early Stage Medical Examination and Treatment and Nursing. Journal of Kyushu for Emergency Medicine, 7, 1-5.

[6] Nakai, N., Nakamura, K. and Sugawara, M. (2015) Emergency Nurses and Their Important Focus of Attention in the Care of Trauma Patients. Journal of Japanese Association for Emergency Nursing, 17, 9-21.

[7] Takeyasu, Y., Sakurai, E., Araki, T., Deguchi, M. and Yomogida, J. (2011) Difficulties Faced by Emergency Nurseshelping Patients and Families in Crisis Situations. Journal of Japanese Association for Emergency Nursing, 13, 1-9.

[8] Takano, S. (2002) Factors Making End-of-Life Care Difficult in the ICU: Analysis of Survey from ICU Nurse. The Japanese Journal of Clinical Research on Death and Dying, 25, 78-84.

[9] Miyazaki, M., Watanabe, Y., Hiramatsu, T., Karakama, S., Minamidate, E. and Kudo, Y. (2016) Current Situations in an ICU and Nurses' Perceptions Regarding Factors Making End-of-Life Care Difficult in the ICU. Journal of Toho Society for Nursing Research, 13, 43-48.

[10] Uesawa, H. and Nakamura, M. (2013) Difficulties of Nurses Who Are Involved in Family's Surrogate Decision-Making for an Emergency Patient and Their Reasons. Journal of Japan Academy of Critical Care Nursing, 9, 6-18.

[11] Harada, R., Yamase, H., Chigira, M. and Hamamoto, J. (2014) Nurse's Emotion for Nursing Practice to the Dying Patients and Their Families in Emergency Room. Bulletin of Tokyo Healthcare University, 9, 9-16.

[12] Suzuki, E., Sugawara, A. and Takahashi, C. (2012) Changes in the Feelings of Emergency Center Nurses in Initial Treatment of Trauma Patients: Considerations for Expressing Emotions through Conversation. Nihon Kango Gakkai Ronbunshu Seijin I , 42, 7-10.

[13] Tatsuno, J., Yamase, H., Takahara, M., Watanabe, Y., Tamura, F., Sato, N., et al. (2008) Survey of Actual Nursing in Emergency Care Settings and Needs for the First Aid Nursing Education Course. Journal of Japanese Association for Emergency 
Nursing, 9, 29-42.

[14] Watanabe, T., Ueno, K. and Kataoka, T. (2014) Emotional Experience of Nurses Involved with the Life and Death of Patients in Emergency Medical Care. Japanese Society of Occupational Medicine and Traumatology, 62, 17-22.

[15] Watanabe, M. and Fujita, S. (2015) Attitudes of Nurses in Supporting the Changing of Gears in Hematopoietic Tumor Patients. Journal of Japanese Society of Cancer Nursing, 29, 7-17.

[16] Tsay, S.L., Halstead, M.T. and McCrone, S. (2001) Predictors of Coping Efficacy, Negative Moods and Post-Traumatic Stress Syndrome Following Major Trauma. International Journal of Nursing Practice, 7, 74-83. https://doi.org/10.1046/j.1440-172X.2001.00257.x

[17] Kigawa, M., Sakuta, H. and Sakaguchi, M. (2005) Team of Dr and Nurse: From the Viewpoint of the Nurse. Emergency Care, 18, 24-30.

[18] Dunn, S. (1992) The Transition from Novice to Comprtrnt Critical Care Nurse. Critical Care Nursing Quarterly, 15, 69-77.

https://doi.org/10.1097/00002727-199205000-00008

[19] Nishikaichi, Y. and Yoshimoto, T. (2019) Predicaments for Nurses Supporting the Families of Terminal Stage Parents in Emergency Care of Intensive Care Units Field: Literature Review. Chiba Academy of Nursing Science, 24, 1-9.

[20] Tamura, Y. and Tsuda, N. (2008) What Is the Reflection? Sonokihontekigainentokango-kangoken kyuuniokeruigi. Nursing Research, 41, 171-181. 